\title{
CARACTERIZACIÓN MORFO AGRONÓMICA DE HÍBRIDOS PROVENIENTES DEL CRUZAMIENTO ENTRE ESPECIES DIPLOIDES DE PAPA
}

\section{MORPHOAGRONOMIC CHARACTERIZATION OF HYBRIDS FROM THE CROSSES AMONG DIPLOID SPECIESOF POTATO}

Oscar Jair Rodríguez Rodríguez • Luis Ernesto Rodríguez Molano 2,4 José Miguel Cotes Torres ${ }^{3}$

\section{RESUMEN}

Se realizó la caracterización morfoagronómica de 2285 genotipos provenientes de 20 familias obtenidas a partir de cruzamientos entre progenitores diploides, cultivados y silvestres de las especies Solanum tuberosum grupo Phureja, Solanum stenotomun subsp. stenotomun, Solanum stenotomun subsp. goniocalyx y Solanum bukasovii. El experimento fue establecido en el municipio de Facatativa (Cundinamarca) y se evaluaron 35 características en cinco etapas del cultivo. Para el registro de los datos se utilizó la guía para las caracterizaciones morfológicas básicas en colecciones de papas nativas del centro internacional de la papa. Al registrarse variables mixtas se utilizó la distancia genética de Gower. Se identificaron cinco grupos principales, en el grupo donde se ubicaron principalmente los cruzamientos entre variedades, se observó un aumento en la cantidad de pares de foliolos laterales, en el grupo donde se ubicó la variedad Guaneña y algunos cruzamientos de S. stenotomun subsp. goniocalix se observaron brotes violeta en forma predominante. Los cruzamientos de S. bukasovii presentaron color de flor lila y/o morado intenso, hábito de crecimiento semiarrosetado y bajos rendimientos. En los cruzamientos de S. stenotomum subsp stenotonum se presentaron tubérculos de piel roja y ojos profundos.

Palabras claves: Caracterización, Variedades, Distancia Gower, Diploides, Dendrograma.

1 Estudiante de Ingeniería Agronómica. Institución: Universidad Nacional Colombia - Sede Bogotá - Facultad de Ciencias Agrarias - Departamento de Agronomía - Calle 45 Carrera 30 - Ciudad Universitaria, Bogotá D.C. - Colombia

2 Profesor Asociado. Institución: Universidad Nacional Colombia - Sede Bogotá - Facultad de Ciencias Agrarias - Departamento de Agronomía - Calle 45 Carrera 30 - Ciudad Universitaria, Bogotá D.C. - Colombia

3 Profesor Asociado. Institución: Universidad Nacional Colombia - Sede Medellín - Facultad de Ciencias Agrarias - Departamento de Ciencias Agronómicas - Cll. 59A No. 63-20 - Núcleo El Volador, Medellín - Colombia

4 Autor para correspondencia: lerodriguezmo@unal.edu.co 


\section{ABSTRACT}

A morphoagronomic characterization of 2285 genotypes from 20 families obtained from crosses of different diploid parents, cultivated and wild belonging to the species Solanum tuberosum group Phureja,Solanum stenotomun subsp. stenotomun, Solanum stenotomun subsp. goniocalyx and Solanum bukasovii was done. An experimental field was established in the municipality of Facatativá (Cundinamarca). 35 Characters were evaluated in five different ages of the crop. For the data record the guide for basic morphological characterizations in native potato collections from the international potato center (CIP) was used. Mixed variables were recorded for that reason was necessary to use the genetic distance of Gower. Five principals groups were found, the group which appear the different crosses among varieties, a rise in the lateral leaflet pair number was observed, in the group where the variety Guaneña and crosses of Solanum stenotomun subsp. goniocalyx appeared, shoots of violet colors were shown in a predominant form. The crosses from S. bukasovii had purple and lilac color flower, growth habit was rossete-like it presented low yields. Among the crosses of S. stenotomum subsp. the tuber skin color was red and the tuber eyes were deep.

Keywords: Characterization, Varieties, Gower Distance, Diploids, Dendrogram.

\section{INTRODUCCIÓN}

En Colombia, el nombre de papa criolla corresponde a los morfotipos que presentan tubérculos con color de piel y carne amarilla (fenotipo yema de huevo) (Rodríguez et al., 2009a). El grupo conformado por las papas criollas está compuesto por un conjunto de variedades nativas que crecen extensamente en los Andes, desde el occidente de Venezuela hasta el centro de Bolivia (Ghislain et al., 2006), con un centro importante de diversidad localizado en el departamento de Nariño. La papas criollas se caracteriza por presentar adaptación a días cortos, brotación en el momento de la cosecha y ser diploides (Huamán y Spooner, 2002). Sin embargo, Ghislain et al., (2006) propone refinar la descripción del grupo Phureja, incluyendo cultivares con ausencia de período de dormancia independiente de su ploidía. Ha sido clasificada como Solanum phureja (Bukasov, 1971; Lechnovich, 1971; Hawkes, 1990; Ochoa, 1990; Ochoa 1999), Solanum tuberosum Grupo Phureja
(Dodds, 1962; Huamán y Spooner, 2002), y recientemente como Solanum tuberosum Grupo Andigenum (Spooner et al., 2007; Rodríguez et al., 2010).

Colombia se considera el primer productor mundial de papa criolla, sembrando al año en promedio 8.500 hectáreas de las cuales se cosechan cerca de 100.000 ton, especialmente en los departamentos de Cundinamarca, Nariño y Boyacá (CNP, 2012; Herrera y Rodríguez, 2012) y exportaciones cercanas a 1000 ton año-1 (Fedepapa, 2012). Esta papa crece entre los 2.000 y 3.000 metros sobre el nivel del mar (msnm), siendo óptima la altura comprendida entre 2.300 y 2.800 msnm (Becerra et al., 2007), con un rango de temperatura entre $10^{\circ} \mathrm{C}$ y $20^{\circ} \mathrm{C}$, en suelos con textura franca y pH de 5,2 a 5,9 (Pérez et al., 2008).

Los genotipos comerciales de papa criolla se destacan por sus cualidades culinarias, agradable sabor, textura harinosa, fácil preparación, y alto potencial de exportación bajo diversas formas de 
procesamiento (Rivera et al., 2006; Rivera et al., 2011). Es de resaltar que en la especie S. phureja se ha reportado importante diversidad en caracteres de importancia como proteína total y materia seca (Rodríguez et al., 2011; Lu et al., 2012), diversidad para caracteres como calidad de frito (Ñústez, 2011); vitaminas ( $\mathrm{C}$ y complejo B), minerales (Fe, Zn, Cu y $\mathrm{Ca}$ ) y carotenoides (Bonierbale et al., 2004; McCord et al., 2012); tolerancia al calor (Gautney y Haynes, 1983), resistencia y/o tolerancia Phytophthora infestans, Ralstonia solanacearum, Spongospora subterranea, Rhizoctonia solani, PVX, PVY y algunos nematodos (Estrada, 2000; Christ y Haynes, 2001; Ramírez et al., 2013; Rendón et al., 2012; Orozco et al., 2012a; Orozco et al., 2012b; Šliwka, et al., 2012); así como al virus del amarillamiento de venas PYV (Vargas, 2010).

Las cultivares nativos son altamente diversos, presentan una gran variabilidad en formas, colores de piel y carne en los tubérculos que no son frecuentes en la variedades mejoradas modernas (Ovchinnikova et al., 2011). La papa cultivada fuera de su centro de origen y diversificación en los Andes de América del Sur, presenta una estrecha base genética, debido originalmente a la limitada introducción de germoplasma a Europa (Lu et al., 2012). La mayoría de las variedades importantes de papa son tetraploides $(2 n=4 x=48)$ y en general susceptibles a plagas y enfermedades que son devastadoras (PGSC, 2011). Razón por la cual las papas silvestres, nativas y cultivadas de los Andes constituyen una base genética importante para mejorar la producción, buscando resistencia a plagas, enfermedades y a diferentes limitantes ambientales y agronómicas (Ovchinnikova et al., 2011).

Dadas las características presentes en especies silvestres como en cultivadas y a la vez la búsqueda de genotipos superiores, los programas de mejoramiento en esta especie permitirían un establecimiento en el mercado nacional y una ampliación del mercado internacional. Pero durante este proceso de mejoramiento, se hace necesario un adecuado registro de variables o caracterización mediante un listado de descriptores cuya expresión sea fácil de medir y que hagan referencia a la forma, estructura o comportamiento de cada accesión (Hidalgo, 2003).

Estos descriptores se pueden clasificar como morfoagronómicos (cualitativos o cuantitativos que pueden proveer indicaciones acerca de rendimiento y pueden llegar a ser afectados por el ambiente) (Vilela de Resende, 2002) y evaluativos (marcadores moleculares, proteínas, isoenzimas y fragmentos de ADN) (Collard et al., 2005). Estos son aplicados en la caracterización y evaluación de accesiones debido a que ayudan a su diferenciación y a expresar un atributo de manera precisa y uniforme, también permiten hacer un uso eficaz de las colecciones y permiten la identificación de genotipos superiores que pueden ser utilizados como progenitores en los programas de mejoramiento. Según Hidalgo (2003) al caracterizar una colección se busca medir la variabilidad de un grupo en estudio, investigar la estructura genética o la forma en la que se compone la colección, identificar posibles duplicados de accesiones (Huamán, 2009) e identificar genes especiales o alelos particulares que se pueden expresar en caracteres visibles.

El objetivo de este trabajo fue realizar la caracterización morfoagronómica de híbridos provenientes del cruzamiento entre especies diploides de papa y procedentes de cruzamientos entre diploides $x$ diploide cultivadas y diploide $x$ silvestre.

\section{MATERIALES Y MÉTODOS}

El trabajo experimental se realizó durante el primer semestre de 2010 en la finca El Porvenir, ubicada a 451'03.32" de latitud norte, 74¹7'56.69" de longitud oeste y una altitud de 2683 msnm., vereda Cuatro Esquinas, municipio de Facatativa, Cundinamarca. La caracterización morfo agronómica se basó en los descriptores propuestos por la "Guía para las caracterizaciones morfológicas básicas en 
colecciones de papas nativas, del Centro Internacional de la Papa CIP" (Gómez, 2000).

Tabla 1. Descriptores morfológicos para la caracterización morfológica evaluados.

\begin{tabular}{|c|c|}
\hline Etapa & Característica \\
\hline Vegetativa & $\begin{array}{l}\text { Vigor } \\
\text { Hábito de crecimiento } \\
\text { Tipo de disección de la hoja } \\
\text { Color de tallo } \\
\text { Forma de alas }\end{array}$ \\
\hline Floración & $\begin{array}{l}\text { Número de folíolos laterales } \\
\text { Número de interhojuelas sobre } \\
\text { peciolulos } \\
\text { Número de interhojuelos entre } \\
\text { folíolos laterales } \\
\text { Grado de floración } \\
\text { Forma de corola } \\
\text { Color predominante de la flor } \\
\text { Intensidad de color predominante } \\
\text { Color secundario de la flor } \\
\text { Distribución del color secundario de } \\
\text { la flor } \\
\text { Pigmentación de anteras } \\
\text { Pigmentación en el pistilo } \\
\text { Color del cáliz } \\
\text { Color del pedicelo }\end{array}$ \\
\hline Fructificación & $\begin{array}{l}\text { Color de la baya } \\
\text { Forma de la baya } \\
\text { Madurez }\end{array}$ \\
\hline Tubérculos & $\begin{array}{l}\text { Color predominante de tubérculo } \\
\text { Intensidad del color predominate } \\
\text { de tubérculo } \\
\text { Color secundario de la piel del } \\
\text { tubérculo } \\
\text { Distribución del color secundario de } \\
\text { la piel del tubérculo } \\
\text { Forma general del tubérculo } \\
\text { Variante de la forma del tubérculo } \\
\text { Profundidad de ojos } \\
\text { Color de la pulpa del tubérculo } \\
\text { Color secundario de la pulpa de } \\
\text { tubérculo } \\
\text { Distribución del color secundario de } \\
\text { la pulpa de tubérculo } \\
\text { Color predominante del brote } \\
\text { Color secundario del brote } \\
\text { Distribución del color secundario } \\
\text { del brote } \\
\text { Rendimiento } \\
\text { Gravedad específica }\end{array}$ \\
\hline
\end{tabular}

Se evaluó un total de 36 características, 30 caracteres cualitativos categóricos correspondientes a los diferentes órganos de la planta y seis cuantitativos, en cinco etapas distintas del cultivo (Tabla 1).

\section{Material vegetal}

Se evaluaron 2285 clones provenientes de 20 familias obtenidas a partir de cruzamientos entre diferentes progenitores diploides, cultivados y silvestres pertenecientes a las especies Solanum tuberosum grupo Phureja, Solanum stenotomun subsp.

Tabla 2. Cruzamientos intra e interespecíficos de familias diploides de papa.

\begin{tabular}{|c|c|}
\hline Familia & $\begin{array}{l}\text { Número de clones } \\
\text { de la progenie }\end{array}$ \\
\hline 98-71-9 x Colombia & 20 \\
\hline Colombia x Galeras & 280 \\
\hline Colombia $\times$ Latina & 190 \\
\hline Colombia x S. stn 703285 & 160 \\
\hline Colombia x S. stn 703315 & 85 \\
\hline Guaneña x Galeras & 65 \\
\hline Guaneña $\times$ Latina & 5 \\
\hline Guaneña x OP & 70 \\
\hline Latina $\times$ S. stn (gon 1) & 45 \\
\hline Latina x UN-col 38 & 230 \\
\hline S. buk $265865-9$ x 98-71-9 & 190 \\
\hline S. buk 473452-12 x Guaneña & 180 \\
\hline S. buk 473452-15 x Guaneña & 125 \\
\hline S. gon $703279 \times$ Colombia & 100 \\
\hline S. gon $703825 \times$ Colombia & 120 \\
\hline S. gon $704481 \times$ Colombia & 155 \\
\hline S. stn 195188-12 x Colombia & 95 \\
\hline $\begin{array}{c}\text { S. stn } 195188-7 \times \text { S. phu } \\
704218\end{array}$ & 20 \\
\hline UN-col $30 \times$ UN-col 62 & 85 \\
\hline UN-col $38 \times$ Galeras & 65 \\
\hline
\end{tabular}


stenotonum, Solanum stenotonum subsp. goniocalyx Juz et Buk y Solanum bukasovii Juz ex Rybin (Tabla 2).

\section{Diseño Experimental}

Se utilizó un diseño incrementado con diez bloques completos al azar, utilizando como testigos cuatro cultivares comerciales (Criolla Colombia, Criolla Guaneña, Criolla Latina y Criolla Galeras) y dos clones avanzados de mejoramiento (98-71-26 y 98-71-9). De cada clon se sembraron cinco tubérculos por surco, a una distancia de siembra de 0,3 m entre plantas y $1 \mathrm{~m}$ entre surcos para una densidad de 33.333 plantas $\cdot$ ha- ${ }^{1}$.

\section{Variables cualitativas}

Cada una de las cinco plantas de cada clon fue observada para posteriormente registrar la moda del carácter, este procedimiento se hizo para las 30 variables evaluadas.

\section{Variables cuantitativas}

Rendimiento de tubérculo: se tomó el peso, el total de la producción, el cual se multiplicó por el número de plantas por parcela y se estimó el rendimiento en kg.planta- ${ }^{1}$.

\section{Gravedad específica}

La variable gravedad específica (GE) se evaluó en el total de la población. Por cada clon se tomó una muestra aleatoria de diez tubérculos sanos y sin residuos de suelo. La variable se evaluó siete días después de la cosecha, utilizando el método propuesto por Talburt y Smith, (1975).

$$
\mathrm{GE}=\frac{\text { Peso en aire }}{\text { Peso en aire-Peso en agua }}(1)
$$

\section{Análisis de datos}

Para las variables cualitativas se consideró la moda (dato más frecuente) para los genotipos dentro de cada cruzamiento, mientras que para las variables cuantitativas, se tomó el promedio de todos los genotipos dentro del mismo cruzamiento. Como se analizaron datos mixtos (cualitativos y cuantitativos) se utilizó la distancia de Gower, método que permite presentar la dispersión de las accesiones o familias en un plano cartesiano o en un dendrograma (Ligarreto, 2003). Para el cálculo de esta distancia se utilizó la ecuación (2), propuesta por Gower (1971) (Gower, 1971; Franco et al., 2005) que considera la similaridad Sij entre dos observaciones iy $j$.

$$
S_{i j}=\frac{\sum_{k=1}^{v} W_{i j k} S_{i j k} \delta_{i j k}}{\sum_{k=1}^{v} W_{i j k} \delta_{i j k}}
$$

donde $\mathrm{S}_{\mathrm{ijk}}$ : el grado de similaridad que se presenta entre dos individuos i y j, para una variable $k$; $\square_{i j k}$ es la posibilidad de efectuar las comparaciones, 1 cuando el carácter $k$ puede ser comparado tanto para i como para j, 0 si no se da la comparación (ausencia de eventos en uno o en los dos individuos) y por último tenemos $W_{i \mathrm{j} k^{\prime}}$ el cual es un peso que se le da a la variable.

Se utilizó el entorno estadístico R (R Development Core Team, 2014) en el cual se generaron programaciones distintas para las variables cuantitativas y cualitativas, asignándole el mismo peso a todas la variables.

Se efectuó el cálculo de la similaridad de forma conjunta, sumando la parte del numerador de las variables cualitativas con el numerador de las variables cuantitativas, luego se realizó el mismo procedimiento con el denominador, posteriormente se calculó el cociente entre la suma del numerador y la suma del denominador (ecuación 3), finalmente se llevó a cabo la generación del dendrograma con la función hclust de R.

$$
S_{i j}=\frac{\sum_{k=1}^{v} W_{i j k a} S_{i j k a} \delta_{i j k a}}{\sum_{k=1}^{v} W_{i j k a} \delta_{i j k a}}+\frac{\sum_{k=1}^{v} W_{i j k b} S_{i j k b} \delta_{i j k b}}{\sum_{k=1}^{v} W_{i j k b} \delta_{i j k b}}
$$


Valores con sub-índices "a" corresponden a las variables cualitativas mientras que los valores con subíndice " $b$ " son los correspondientes a las variables cuantitativas.

\section{RESULTADOS Y DISCUSIÓN}

Las variables tipo de disección de la hoja (disectada), pigmentación en anteras (sin antocianinas) y pigmentación en el pistilo (sin antocianinas), no presentaron variación razón por la cual fueron excluidas del análisis por no presentar poder discriminatorio. Este resultado coincide con el reportado por Bernal et al., (2006) cuando realizó la detección de posibles duplicados en la Colección Central Colombiana de papa Solanum tuberosum subsp. andigena a partir

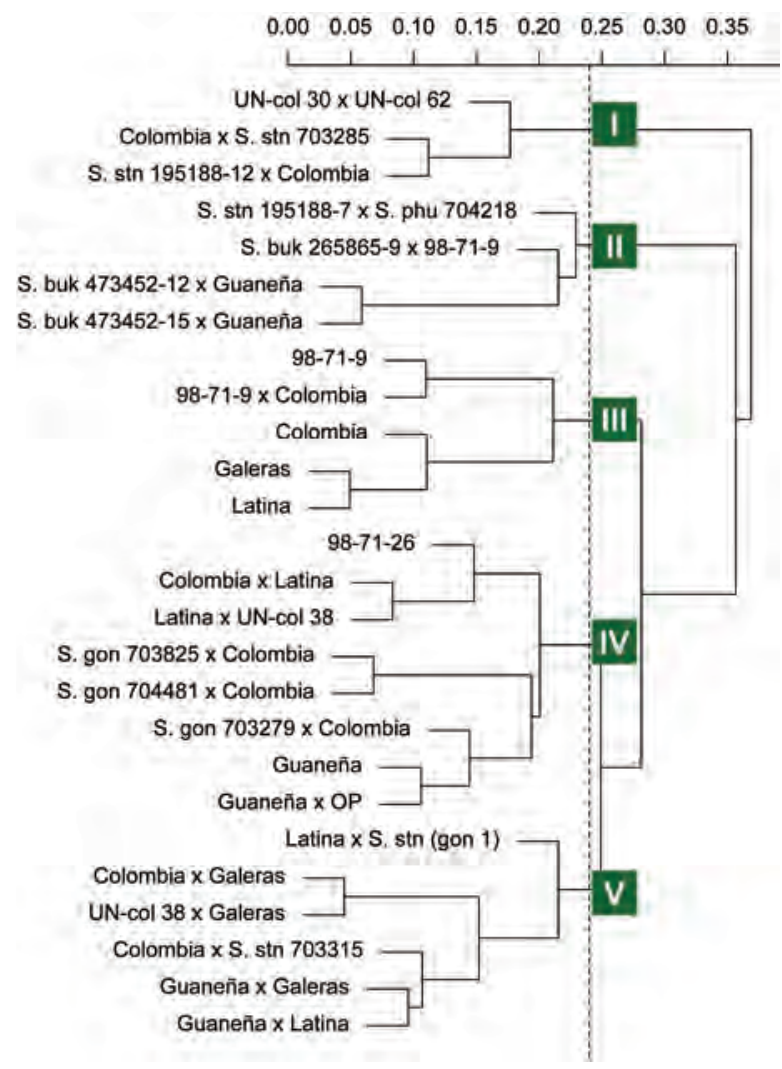

Figura 1. Dendrograma de las 20 familias y seis clones en estudio. En el eje horizontal está el coeficiente de disimilitud de Gower. En verde se destacan los brazos del dendrograma que conforman los grupos escogidos. de caracteres morfológicos. Al obtener los resultados del dendrograma para los cruzamientos dentro del análisis se puede ver la conformación de cinco grupos principales (Figura 1).

\section{Grupo I}

En este grupo se encontraron la mayoría de cruzamientos de diferentes cultivares con la especie $S$. stenotonum subsp stenotonum. Presentaron hábito de crecimiento semierecto, colores de tallo verde con pocas manchas y forma de las alas del tallo ondulada en la mayoría de los casos con excepción de UN-col 30 x UNcol62 donde fue recta. El número de pares de folíolos laterales y de interhojuelas entre folíolos laterales fue de cinco. En todos los cruzamientos se presentó al menos un par de interhojuelas sobre peciolulos.

El grado de floración fue moderado, la forma de la corola para Criolla Colombia x S. stn 703285 y S. stn 195188-12 x Criolla Colombia fue rotada y para los demás cruzamientos fue pentagonal., El color predominante de flor fue violeta con intensidad intermedia excepto en el cruzamiento UNcol $30 \mathrm{x}$ UNcol62 en el que predominaron las coloraciones blancas de intensidad intermedia, no se presentó coloración secundaria en ninguno de los cruzamientos. El color de cáliz en todos los casos fue verde con abundantes manchas y el color de pedicelo presentó bastantes variaciones, para UNcol 30 x UNcol62 pigmentado con articulación verde, Criolla Colombia $x$ S. stn 703285 fue ligeramente pigmentado y para S. stn 195188-12 x Criolla Colombia no presentó algún tipo de coloración diferente a la verde.

El color de la baya en la mayoría de los casos fue verde excepto en el cruzamiento Criolla Colombia $x$ S. stn 703285, la forma de la baya fue predominantemente ovoide. En este grupo predominan los colores de piel de tubérculo rojos intensos, característica considerada por Ligarreto et al., (1997) como una de las más significativas al momento de caracterizar 
colecciones de papa. Ésta se puede observar principalmente en los cruzamientos Criolla Colombia $x$ S. stn 703285 y S. stn 195188-12 x Criolla Colombia, mientras que el cruzamiento UN-col30 x UN-col62 fue amarillo intenso.

La forma general del tubérculo fue redonda con excepción del cruzamiento UNcol30 x UNcol 62 donde predominaron las formas ovaladas, la profundidad de ojos en la mayoría de los cruzamientos fue media, no obstante en Criolla Colombia x S. stn 703285 predominaron los ojos profundos. El color de carne del tubérculo presentó diferentes tonalidades de amarillo especialmente en los cruzamientos $S$. stn 195188 x Criolla Colombia y Criolla Colombiax S. stn 703285 donde para el primer cruzamiento la tonalidad fue amarilla, para los cruzamientos restantes la tonalidad del amarillo fue intenso, para los cruzamientos UNcol30 x UNcol62 y Criolla Colombia x $S$. stn 703285, las tonalidades fueron crema. Las coloraciones del brote en todos los cruzamientos fueron rojas con colores secundarios blancos distribuidos en las yemas.

Los valores de producción por planta variaron entre $3.069 \mathrm{~g}$ (UN-col30 y UN-col62) y $2.084 \mathrm{~g}$ (Criolla Colombia x S. stn 703285), los valores de gravedad específica oscilaron entre 1,080 y 1,087.

\section{Grupo II}

En el grupo se ubican los individuos provenientes del cruzamiento entre S. stn 195188-7 x S. phu 704218 y todos los cruzamientos donde se utilizó la especie silvestre S. bukasovii. La mayoría de las plantas presentaron porte bajo a medio, crecimiento arrosetado y menor vigor que el observado para los cruzamientos entre especies diploides cultivadas, pero un porte muy superior al observado para el progenitor silvestre. El número de pares de folíolos laterales fue mayor en estas familias con un promedio de cinco, mientras que el número de interhojuelas entre folíolos laterales fue de cuatro pares, además en todos los casos se presentó al menos un par de interhojuelas sobre peciolulos. Esta condición es mayor en las hojas de las especies silvestres manifestando algún grado de dominancia.

El hábito de crecimiento para los que presentaron como parental al cultivar Criolla Guaneña fue semiarrosetado, en los dos cruzamientos restantes, fueron semierectos. El color del tallo presentó tonalidades verdes con pocas manchas, la forma de las alas del tallo para todos los cruzamientos fue predominantemente ondulada, el grado de floración fue moderado y la forma de la corola varío entre pentagonal para S. buk 473452-12 x Criolla Guaneña y S. buk $473452-15 \times$ Criolla Guaneña y rotada para S. buk $265865-9$ × 98-71-9 y S. stn 195188-7 x S. phu 704218.

Se observaron diferencias marcadas en términos de la intensidad del color predominante de flor con respecto a los demás grupos ya que el violeta fue mucho más intenso. El cruzamiento S. buk 265865$9 \times$ 98-71-9 presentó coloración de flor lila pero de igual manera con tonalidades intensas, tampoco se presentaron colores secundarios en flor, los colores de cáliz fueron pigmentados en todos los cruzamientos donde se encontraba la especie $S$. bukasovii, mientras el cruzamiento S. stn 195188-7 × S. phu 704218 presentó pocas manchas. El color de pedicelo en los cruzamientos que tenían progenitor al cultivar Criolla Guaneña como parental fue mayormente pigmentado con articulación verde, mientras que en los demás cruzamientos sólo se dio una pigmentación ligera del pedicelo.

Los cruzamientos que presentaron como parental a Criolla Guaneña tuvieron una baya de color verde con áreas pigmentadas, mientras para $S$. buk 265865-9 x 98-71-9 el color de la baya fue verde con abundantes puntos blancos y S. stn 195188-7 x S. phu 704218 presentó únicamente tonalidades verdes; de igual manera la forma de la baya presentó variaciones entre globosa para los cruzamientos $S$. buk $265865-9$ × 98-71-9 y S. stn 195188-7 x S. phu 
704218 y ovoide, para los casos cuyo parental fue la variedad Criolla Guaneña.

En el color predominante de piel de tubérculo se observan marcadas diferencias con respecto a los demás grupos, ya que se encuentran tonalidades naranja con intensidad pálida para S. buk 2658659 x 98-71-9 y S. buk 473452-15 x Criolla Guaneña, amarillo intenso para S. stn 195188-7 x S. phu 704218 y blanco crema intenso para el cruzamiento $S$. buk 473452-12 x Criolla Guaneña. La forma general del tubérculo fue comprimida para S. buk 473452-12 x Criolla Guaneña y S. stn 195188-7 x S. phu 704218, redonda para S. buk 473452-15 x Criolla Guaneña y obovado en el caso de S. buk 265865-9 x 98-71-9, así mismo, los ojos del tubérculo tuvieron tendencias a ser más superficiales en S. buk 265865-9 x 98-71-9 y S. buk 473452-15 x Criolla Guaneña y con profundidad moderada en los demás casos. El color de carne de tubérculo predominante en la mayoría de los cruzamientos fue crema; en el rasgo color de brote se presentaron colores primarios violeta y color secundario blanco distribuido principalmente en las yemas para todos los casos, excepto el cruzamiento S. stn 195188-7 x S. phu 704218 con tonalidades rojas pero con el mismo color secundario y distribución.

Este grupo presentó valores de producción por planta inferiores a $1.370 \mathrm{~g}$, siendo los más bajos de todos los grupos. Los valores de gravedad específica fueron mayores a 1,090, debido posiblemente a que uno de los progenitores de los cruzamientos es la variedad Criolla Guaneña presentó valores de gravedad específica cercanos 1,107 $\pm 0,0001$ en trabajos realizados por Herrera et al., (2010).

\section{Grupo III}

El hábito de crecimiento para todos los individuos fue semierecto, con color del tallo verde, con forma onduladas para las alas del tallo, excepto para la variedad Criolla Latina que presentó alas del tallo de forma recta. La variable número de folíolos laterales presentó un promedio de tres pares de foliolos, observándose un par de interhojuelas entre foliolos laterales. Ninguna de las familias del grupo presentó interhojuelas sobre peciolulos.

La familia proveniente del cruzamiento 98-71-9 x Criolla Colombia, presentó floración escasa, mientras que los cultivares restantes presentaron un grado de floración moderada, la forma de la corola varió entre pentagonal y rotada, el color principal de la flor fue violeta con una intensidad intermedia, no obstante, se presentó coloración de flor secundaria blanca para la familia 98-71-9 x Criolla Colombia y en los cultivares Criolla Colombia y Criolla Latina, en el primero distribuyéndose tanto en el haz como en el envés mientras en las dos últimas únicamente se presentó esta coloración en el envés.

El color del cáliz en la mayoría de los casos fue verde con pocas manchas, en el único caso donde se

\section{Todas las familias que tuvieron como parental a S. stenotonum subsp. goniocalyx presentaron gravedades específicas superio- res a 1.090 siendo de gran interés para la industria del procesa- miento de papa.}


presentó cáliz de color verde con abundantes manchas fue en la familia 98-71-9 x Criolla Colombia, una tendencia similar se observó en la variable color de pedicelo donde este cruzamiento fue el único que presentó ligeras pigmentaciones a lo largo y en la articulación del pedicelo, mientras para los demás fue únicamente verde.

El color de la baya en todos los casos fue verde con pocos puntos blancos, la forma de la baya varió entre globosa y ovoide, esta última se presentó con mayor frecuencia en Criolla Colombia. Para la variable color principal de la piel del tubérculo todas presentaron tonalidades amarillas con intensidad intermedia, la intensidad del color fue mayor en la familia 98-71-9. La forma general del tubérculo fue redonda y la profundidad de ojos del tubérculo fue intermedia. Se observaron tonalidades amarillas intensas para el color de carne del tubérculo en todos los cruzamientos. Los colores de brotes fueron rojos, difiriendo con lo citado por Rodríguez et al., (2009) quienes consideran que cultivares como Criolla Latina y Criolla Colombia presentan colores de brote lila. Estos brotes presentaron color secundario blanco ubicado principalmente en las yemas.

En este grupo los cultivares comerciales presentaron una producción por planta que osciló entre 2.810 y 3.266 g. El genotipo avanzados 98-71-9, presentó el menor rendimiento con valores de $1.187 \mathrm{~g}$. Para la variable gravedad específica los tubérculos provenientes del cultivar Criolla Colombia presentaron el mayor valor con un promedio de 1,093, ratificando los observado por Rodríguez et al., (2009) al caracterizarse este cultivar por presentar altos contenido demateria seca, excelente calidad culinaria con características excepcionales para consumo fresco o procesamiento en la presentación precocido congelado.

\section{Grupo IV}

Es uno de los grupos más amplios, es decir, que comprende la mayor cantidad de familias, en el cual se encuentran todos los cruzamientos con S.stenotonum subsp goniocalyx. Poseen hábito de crecimiento semierecto, con color del tallo es dominante verde y presentaron alas, excepto la familia S. gon 703279 x Criolla Colombia que mostró leves manchas en el taIlo. El número de pares de folíolos laterales fue de cinco para los cruzamientos S. gon 703825 x Colombia y S. gon 704481 x Colombia, y de cuatro para el resto de familias, mientras que el número de interhojuelas entre folíolos laterales fue de uno para los que tuvieron como parental a Criolla Guaneña y de tres para el resto de los miembros del grupo. En general los individuos del grupo se caracterizan por no presentar interhojuelas sobre peciolulos.

El grupo tuvo una moderada floración, siendo que la flor posee una corola pentagonal en la mayoría de familias del grupo, es de color violeta intenso y sin color secundario. El cáliz fue de color verde con manchas, el pedicelo de color verde, excepto para la familia S. gon 703279 x Criolla Colombia, el cual fue bastante pigmentado. Los frutos variaron entre verdes y verdes con puntos blancos, de forma ovoide y de maduración intermedia, con excepción del clon 98-71-26 que presentó una maduración precoz.

Los tubérculos presentaron piel de color anaranjado y su intensidad varió de pálido a intermedio. Hubo ausencia de color secundario en la piel del tubérculo. En cuanto al color de la pulpa del tubérculo el grupo fue bastante diverso, la familia Criolla Latina x UN-col 38 y el clon 98-71-26 presentó color amarillo intenso, la familia Criolla Guaneña x OP fue de color crema, y los restantes miembros del grupo fueron de color amarillo. En todo el grupo hubo ausencia de color secundario de la pulpa. La forma del tubérculo fue comprimida en las familias que tienen como parental a S. stenotonum subsp. goniocalyx, mientras que en el resto del grupo fueron redondos. El grupo presentó tubérculos con ojos poco profundos y los brotes fueron predominantemente rojos sin color secundario. 
El rendimiento del grupo osciló entre 1.187 y 2.415 g planta- para el clon 98-71-26 y la familia Criolla Guaneña x OP, respectivamente. Exceptuando la familia Criolla Colombia x Criolla Latina, que presentó una gravedad específica muy baja $(1,077)$, los demás miembros del grupo tienen una potencial para la industria de chips presentando valores superiores a 1,080 (Pérez et al., 2008). Todas las familias que tuvieron como parental a S. stenotonum subsp. goniocalyx presentaron gravedades específicas superiores a 1,090 siendo estos híbridos interespecíficos de gran interés para la industria del procesamiento de papa.

\section{Grupo V}

En este grupo se encuentra los cruzamientos cuyo parental es Criolla Galeras. Se caracteriza por ser un grupo de hábito de crecimiento semierecto y color de tallo verde. Todas la familias dentro del grupo presentaron un promedio de cinco folíolos, y el número de interhojuelas por folíolo fue diverso, siendo dos para las familias Criolla Colombia x Criolla Galeras y UN-col $38 \times$ Criolla Galeras, tres en los cruzamientos Criolla Colombia x S. stn 703315 y Criolla Guaneña x Criolla Galeras, y cuatro en los restantes miembros del grupo. En general los individuos del grupo se caracterizan por no presentar interhojuelas sobre peciolulos.

La floración fue moderada, y las flores fueron de color violeta con intensidad del color intermedia y sin color secundario. La forma de la corola fue pentagonal para los cruzamientos UN-col 38 x Criolla Galeras y Criolla Colombia x Criolla Galeras, mientras que para el resto del grupo fue rotada. El color del cáliz fue verde con pocas manchas para los cruzamientos Criolla Guaneña x Criolla Galeras y Criolla Guaneña x Criolla Latina, mientras que para el resto del grupo fue verde con manchas abundantes. La baya fue de color verde, forma globosa y maduró de los 120 a los 149 días.

Los tubérculos fueron redondos, de color de piel anaranjado, exceptuando el cruzamiento Colombia x S. stn 703315 con piel amarilla, el color de la pulpa puede ser crema, amarillo o amarillo profundo. Los tubérculos no presentaron color secundario en la pulpa o en la piel. En cuanto a la profundidad de los ojos fue media exceptuando el cruzamiento UN-col $38 \times$ Criolla Galeras donde los ojos son profundos. El color del brote puede ser rojo o violeta y no presentaron color secundario.

La producción por planta osciló entre 1.781 y 2.504 g, para los cruzamientos Latina $\times$ S. stn (gon 1) y Criolla Guaneña x Criolla Galeras, respectivamente. La GE varió muy poco en el grupo siendo menor la del cruzamiento Criolla Guaneña x Criolla Latina $(1,074)$ y la mayor la presentada por la familia Criolla Colombia x Criolla Galeras $(1,088)$. El cruzamiento Criolla Guaneña x Criolla Latina no tiene aptitud industrial ya que valores inferiores a 1,078 son penalizados por la industria (Guenthner, 2001; Palacios et al., 2008), sin embargo los demás miembros del grupo presentan valores adecuados de este parámetro industrial.

\section{AGRADECIMIENTOS}

Esta investigación fue financiada a través del Convenio: 2007S7647, Contrato: IV 1344, cofinanciado por el Ministerio de Agricultura y Desarrollo Rural,el Fondo Nacional Hortofrutícola, la Federación de Productores de Papa, FEDEPAPA y la Universidad Nacional de Colombia. Los autores expresan sus agradecimientos a los integrantes del Grupo de Investigación en papa (COL0010065), que aportaron el recurso humano para desarrollar esta investigación. 


\section{BIBLIOGRAFÍA}

1. Becerra L., Navia S., Nústez C. 2007. Efecto de niveles de fósforo y potasio sobre el rendimiento del cultivar 'Criolla Guaneña' en el departamento de Nariño. Revista Latinoamericana de la Papa14: 51-60.

2. Bernal A., Arias J., Moreno J., Valbuena I., Rodríguez L. 2006. Detección de posibles duplicados en la Colección Central Colombiana de papa Solanum tuberosum subespecie andigena a partir de caracteres morfológicos. Agronomía Colombiana 24: 226 - 237.

3. Bonierbale M., Amoros W., Espinoza J., Mihovilovich E., Roca W., Gómez R. 2004. Recursos genéticos de la papa: don del pasado, legado para el futuro. Suplemento revista Latinoamericana de la papa 1: 3 -13.

4. Bukasov S.M. 1971. Cultivated potato species. pp. 5-40. En: Bukasov, S.M. (ed.). Flora of cultivated plants. Vol. IX. Kolos, Leningrad, Rusia.

5. Christ B.J., Haynes K.G. 2001. Inheritance of resistance to early blight disease in a diploid potato population. Plant Breed 120:169-172.

6. CNP. 2012. Acuerdo de competitividad de la cadena agroalimentaria de la papa en Colombia. Diciembre 2010. Consejo Nacional de la Papa, Bogotá D.C., Colombia, 72 p. http://www.fedepapa.com/wp-content/uploads/pdf/ACUERDO-COMPETITIVIDAD-CADENA-AGROALIMENTARIA-PAPA.pdf consulta en Enero 2014.

7. Collard B., Jahufer M., Brouwer J., Pang E. 2005. An introduction to markers, quantitative trait loci (QTL) mapping and marker-assisted selection for crop improvement: The basic concepts. Euphytica 142: 169-196.
8. Dodds K.S. 1962. Classification of cultivatedpotatoes. pp. 517-539. En: Correll, D.S. (ed.). The potato and its wild relatives. Series of Botanical Studies 4, Texas Research Foundation, Renner (TX), USA, 606p.

9. Estrada N. 2000. La biodiversidad en el mejoramiento genético de la papa. PROINPA. La Paz, Bolivia, $199 \mathrm{p}$.

10. FEDEPAPA. 2012. Indicadores. En: Revista Papa. Fedepapa: No: 25: 45 p.

11. Franco J., Crossa J., Suketoshi T., Shands H. 2005. A Sampling Strategy for Conserving Genetic Diversity when Forming Core Subsets. Crop Science 45: 1035-1044.

12. Gautney T.L., Haynes F.L. 1983. Recurrent selection for heat tolerance in diploid potatoes (Solanum tuberosum subspp. phureja and stenotomum). American Potato Journal 60: 537-5452.

13. Ghislain M., Andrade D., Rodríguez F., Hijmans R.J., Spooner D.M. 2006. Genetic analysis of the cultivated potato Solanun tuberosum L. Phureja Group using RAPDs and nuclear SSRs. Theoreticaland Applied Genetics 113: 1515-1527.

14. Gómez R. 2000. Centro Internacional de la Papa (CIP). Guía para las Caracterizaciones Morfológicas Básicas en Colecciones de Papas Nativas. Centro Internacional de la papa (CIP), Lima, Perú, 27 p.

15. Hawkes J.G. 1990. The potato: evolution, biodiversity and genetic resources. Belhaven Press, London UK, 259p. 
16. Herrera A.O., Rodríguez L.E. 2012. Tecnologías de producción y transformación de papa criolla. Universidad Nacional de Colombia. Facultad de Agronomía, Bogotá D.C. Colombia.120 p.

17. Hidalgo R. 2003. Variabilidad genética y caracterización de especies vegetales. Análisis Estadístico de datos de caracterización morfológica de recursos fitogenéticos. Boletín técnico IPGRI 8: 2 - 27.

18. Huamán Z. 2009. Descriptores morfológicos de la papa (Solanum tuberosum L). Centro de Conservación de la Biodiversidad Agrícola de Tenerife CCBAT. Cabildo de Tenerife, España, 36 p.

19. Huamán Z., Spooner D.M. 2002. Reclassification of landrace populations of cultivated potatoes (Solanum sect. Petota). American Journal of Botany 89: 947-965.

20. Lechnovich V.S. 1971. Cultivated potato species. pp. 41-304. En: Bukasov, S.M. (ed.). Flora of cultivated plants. Vol. IX. Kolos, Leningrad, Rusia.

21. Ligarreto G. 2003. Análisis de la variabilidad genética en fríjol. Análisis Estadístico de datos de caracterización morfológica de recursos fitogenéticos. Boletín técnico IPGRI 8: 40 - 50.

22. Ligarreto G., Suárez M. 2003. Evaluación del potencial de los recursos genéticos de papa criolla (Solanum phureja) por calidad industrial., Agronomía Colombiana 21: 83-94.

23. Ligarreto G., Valbuena I., Fajardo D. 1997. Caracterización morfológica de la colección central colombiana de papa. En: Memorias V Congreso Sociedad Colombiana de Fitomejoramiento y producción de cultivos. Santa Marta, Colombia.
24. Lu W., Yu M., Bai Y., Li W., Xu X. 2012. Crude Protein Content in Diploid Hybrid Potato Clones of Solanum phureja-S. stenotomum . Potato Research 55: 315-322.

25. Mc Gregor I. 2007. The fresh potato market. pp. 3 - 26. En: Vreugdenhil D., Bradshaw J., Gebhardt C., Govers F., MacKerron D., Taylor M., Ross H. (eds.). Potato biology and biotechnology. Elsevier Ltd., London, UK, 857 p.

26. McCord P., Zhang L., Brown C. 2012. The Incidence and Effect on Total Tuber Carotenoids of a Recessive Zeaxanthin Epoxidase Allele (Zep1) in Yellow-fleshed Potatoes. American Journal of Potato Research 89: 262-268.

27. Nústez, C. E. 2011. Estudios fenotípicos y genéticos asociados a la calidad de fritura en Solanum phureja Juz et Buk. Tesis de Doctorado en Ciencias Agrarias, Facultad de Agronomía, Universidad Nacional de Colombia Sede Bogotá, Bogotá D.C., 202 p.

28. Ochoa C.M. 1990. The potatoes of South America: Bolivia. Cambridge University Press, Cambridge, UK, $512 \mathrm{p}$.

29. Ochoa C.M. 1999. Las papas de Sudamérica: Perú. Allen Press, Lawrence (KS), USA, 1036p.

30. Orozco L.F., Zuluaga C.M., Cotes J.M. 2012a. Selección combinada en una población de Solanum phureja para resistencia a Spongospora subterranea f. sp. subterranea y Phytophthora infestans. Revista Facultad de Ciencias Básicas 8: $56-60$.

31. Orozco L.F., Zuluaga C.M. y Cotes J.M. 2012b. Ganancia genética esperada de la resistencia a sarna polvolsa en una población de papa 
criolla. Revista Facultad de Ciencias Básicas 8: $210-225$.

32. Ovchinnikova A., Krylova E., Gavrilenko T., Smekalova T., Zhuk M., Knapp S., Spooner D.M. 2011. Taxonomy of cultivated potatoes (Solanum section Petota: Solanaceae). Botanical Journal of the Linnean Society 165: 107-155.

33. Palacios, C., Jaramillo S., González L., Cotes J.M. 2008. Efecto de la fertilización sobre la calidad de la papa para procesamiento en dos suelos antioqueños con propiedades ándicas. Agronomía Colombiana 26: 487-496.

34. Pérez, L., Rodríguez L., Gómez M. 2008. Efecto del fraccionamiento de la fertilización con N, $\mathrm{P}$, K y Mg y la aplicación de los micronutrientes $B$, Mn y $\mathrm{Zn}$ en el rendimiento y calidad de papa criolla (Solanum phureja) variedad Criolla Colombia. Agronomía Colombiana 26: 477-486.

35. Pérez L., Rodríguez L.E., Gómez M. 2008. Efecto del fraccionamiento de la fertilización con $\mathrm{N}$, $\mathrm{P}, \mathrm{K}$ y Mg y la aplicación de los micronutrientes $\mathrm{B}, \mathrm{Mn}$ y $\mathrm{Zn}$ en el rendimiento y calidad de papa criolla (Solanum phureja) variedad "Criolla Colombia". Agronomía Colombiana 26: 477-485.

36. PGSC. 2011. Genome sequence and analysis of the tuber crop potato. Nature 475: 189-197.

37. R Development Core Team. 2014. R: A Language and Environment for Statistical Computing. R Foundation for Statistical Computing.
Vienna,Austria. http://www.R-project.org/ consulta en enero 2014

38. Ramírez L.A., González E.P., Zuluaga C.M., Marín M.A., Morales J.G., Nústez C.E., Cotes J.M.2013. Uso de esquejes de tallo lateral para evaluar la resistencia de genotipos de Solanum phureja a Spongospora subterranea f. sp. subterranea. Tropical Plant Pathology 38: 303 - 312.

39. Rendón V.A., González E.P., Cotes J.M.2012. Heredabilidad de la resistencia a Spongospora subterraneaf. sp. subterranea en una población de Solanum phureja a través de bioensayos. Revista Facultad de Ciencias Básicas 8: 70 - 87.

40. Rivera J., Herrera A., Rodríguez L.E. 2006. Evaluación sensorial en productos procesados de papa criolla (Solanum phureja) y su importancia para el fitomejoramiento. Fitotecnia colombiana 6: 9-25.

41. Rivera J., Herrera A., Rodríguez L.E. 2011. Assessment of the processing profile of six genotypes of "Creole Potato" (Solanum tuberosum Phureja Group). Agronomía Colombiana 29: 47-53.

42. Rodríguez D., Nústez C.E., Cotes J.M., Rodríguez L.E. 2011. Heredabilidad del contenido de proteína total en papa diploide Solanum tuberosum grupo Phureja. Bragantia 70: 759-766.

43. Rodríguez F., M. Ghislain, Clausen A.M., Jansky S.H., Spooner D.M. 2010. Hybrid origins of 
cultivated potatoes. Theoretical and Applied Genetics 121: 1187-1198.

44. Rodríguez, L. 2010. Origen y evolución de la papa cultivada. Una revisión. Agronomía Colombiana 28: 9 - 18.

45. Rodríguez L.E, Nústez C.E., Estrada N. 2009. Criolla Latina, Criolla Paisa y Criolla Colombia, nuevos cultivares de papa criolla para el departamento de Antioquia (Colombia). Agronomía Colombiana 27: 289 - 303.

46. Spooner D.M., Núñez J., Trujillo G., Herrera M.R., Guzmán F., Ghislain M. 2007. Extensive simple sequence repeat genotyping of potato landraces supports a major reevaluation of their gene pool structure and classification. PNAS 104: 19398-19403.

47. Talburt W.F., Smith O. 1975. The potato of processing. The AVI Publishing, Inc. Westport, Connecticut, USA, 705 p.

48. Vargas A.M. 2010. Respuesta de la colección central colombiana de Solanum phureja de la Universidad Nacional de Colombia a la infección con Potato Yellow Vein Virus (PYVV). Tesis de Maestría en Ciencias Agrarias - Énfasis en Fitopatología, Facultad de Agronomía, Universidad Nacional de Colombia. 90 p.

49. Vilela de Resende, 2002. Genética biométrica e estatística no melhoramento de plantas perenes. Embrapa informação científica, Brasilia. Brasil, $975 \mathrm{p}$. 